\title{
On the nature of the inter-micellar interactions in ethoxylated polysorbate surfactants with high degrees of ethoxylation
}

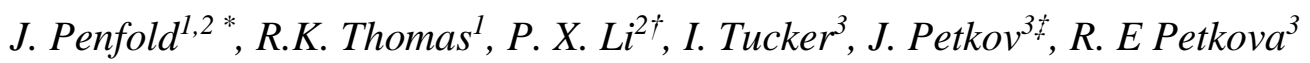

1. Physical and Theoretical Chemistry Laboratory, Oxford University, South Parks Road, Oxford, OX1 3QZ, UK

2. ISIS, STFC, Rutherford Appleton Laboratory, Chilton, Didcot, Oxon, OX11 0QX, UK

3. Unilever Research and Development Laboratory, Port Sunlight, Quarry Road East, Bebington, Wirral, CH63 2JW, UK

RECEIVED DATE (to be automatically inserted after your manuscript is accepted if required according to the journal that you are submitting your paper to)

Keywords: ethoxylated polysorbate surfactants, self-assembly, micelles, inter-micellar interactions, small angle neutron scattering, zeta potential

$†$ Current address: STFC, Rutherford Appleton Laboratory, Harwell, Didcot, OXON, OX1 0QX, UK

+ Current address: KLK Oleo, SDN BHD, Menara KLK, Mutiara Damansara, 47810 Petaling, Jaya Selanger, Malaysia

* Corresponding Author: Jeff Penfold, Phone: +44 1235 445681, Email: jeff.penfold@stfc.ac.uk 


\begin{abstract}
The ethoxylated polysorbate, Tween, nonionic surfactants are extensively used as foam and emulsion stabilisers, and in aqueous solution form globular micelles. The ethoxylated polysorbate surfactants with higher degrees of ethoxylation than the Tween surfactants exhibit some interesting self-assembly properties. Small angle neutron scattering, SANS, measurements have revealed inter-micellar interactions which are more pronounced than the hard-sphere excluded volume interactions normally associated with nonionic surfactant micelles. The interactions are interpreted as arising from the partial charge on the ether oxygen of the ethylene oxide groups. This gives rise to an effective net negative charge on the micelles, which has been determined from the SANS data and zeta potential measurements. For degrees of ethoxylation of twenty and less the effect is relatively small. The interaction increases with increasing ethoxylation, such that for a degree of ethoxylation of fifty the interaction is comparable to that of ionic surfactant micelles. Unlike the intermicellar interaction in ionic surfactant micellar solutions, which result from the charge on the micelle arising from the partial binding of counterions, the interaction between ethoxthylated polysorbate surfactant micelles is unaffected by the addition of electrolyte.
\end{abstract}




\section{INTRODUCTION}

Nonionic surfactants are an important class of surfactants which have been extensively studied (1) and characterised (2); and are predominantly in the form of alkyl polyethylene glycol ethers $\left(\mathrm{C}_{\mathrm{n}} \mathrm{E}_{\mathrm{m}}\right.$, where $\mathrm{n}$ is the alkyl chain length and $\mathrm{m}$ is the number of ethylene oxide groups) and alkyl phenol poly (ethylene oxide) ethers (2). The Tween surfactants are biocompatible nonionic surfactants which are ethoxylated sorbitan esters of different fatty acids $(3,4)$. The Tween have relatively low critical micellar concentrations, cmc, $\sim 10$ to $50 \mu \mathrm{M}$, and HLB, hydrophilelipophile balance, values 15-17; and are ideal as emulsifiers, detergents and solubiliser in foods $(5,6)$, cosmetics (7), and pharmaceuticals (8-10). The commercially available Tweens have predominantly twenty ethylene oxide groups attached to the sorbitan headgroup, with different long chain saturated carboxylic acids, from Lauric to Stearic, and unsaturated Oleic. Recently Penfold et al (11) have investigated the ethoxylated polysorbate nonionic surfactants with a wider range of ethoxylation, from three to fifty ethylene oxide groups. This has resulted in a potentially interesting range of adsorption and self-assembly properties (11), and in the formation of surface multilayer structures when combined with the protein hydrophobin (12).

The self-assembly in aqueous solution of a wide range of nonionic surfactants (13-23), and pluronic block co-polymers (24-26) has been studied by scattering techniques, such as small angle neutron scattering, SANS, small angle x-ray scattering, SAXS, and light scattering. At relatively low surfactant concentrations the nonionic surfactants self-assemble predominantly as globular micelles (13-20). The micelles are treated as uncharged, and overwhelmingly the structure factor, $\mathrm{S}(\mathrm{Q})$, arising from inter-micellar interactions, is consistent with a hard-sphere interaction. The exception to this is the situation at elevated temperatures, as the lower consolute boundary of the surfactant is approached, where the onset of attractive interactions has been modelled by a variety of approaches $(13,14,17,18)$. Some limited evidence for an intermicellar interaction greater than just arising from excluded volume (hard-sphere) for $\mathrm{C}_{12} \mathrm{E}_{8}$ was presented (18), but its origin was not discussed. From studies of the interaction of poly(ethylene oxide) with ionic surfactants (27) and the adsorption of poly(ethylene oxide) onto mica (28), the role of the partial charge on the ether oxygen group was implicated. This raises the possibility that a measurable contribution to the inter-micellar interactions in nonionic surfactants could be electrostatic if the degree of ethoxylation was sufficiently large. However, it has not generally been observed in $\mathrm{C}_{12} \mathrm{E}_{8}$, and $\mathrm{C}_{12} \mathrm{E}_{12}$ micelles $(19,20)$, in Triton X-100 and Brij35 nonionic surfactants, with degrees of ethoxylation of 10 and 23 respectively $(22,23)$. Notably it has also 
not been observed in the Pluronic block copolymer micelles of $\mathrm{EO}_{25} \mathrm{PO}_{40} \mathrm{EO}_{25}, \mathrm{EO}_{37} \mathrm{PO}_{56} \mathrm{EO}_{37}$, $\mathrm{EO}_{67} \mathrm{PO}_{38} \mathrm{EO}_{67}, \mathrm{EO}_{96} \mathrm{PO}_{39} \mathrm{EO}_{96}$ and $\mathrm{EO}_{99} \mathrm{PO}_{65} \mathrm{EO}_{99}$ (where $\mathrm{EO}$ and $\mathrm{PO}$ refer to ethylene oxide and propylene oxide respectively) (24-26), which have relatively large degrees of ethoxylation.

The self-assembly of the Tween-like ethoxylated polysorbate surfactants have been reported for lauric and stearic alkyl chains and degrees of ethoxylation of three to twenty (11). For both the lauric and stearic alkyl chains and degrees of ethoxylation $<13$ the self-assembly is predominantly lamellar. For degrees of ethoxylation > 13 globular micelles form, and at surfactant concentrations in the range 5 to $20 \mathrm{mM}$ the inter-micellar interactions were adequately described as hard sphere.

In this paper we explore the nature of inter-micellar interactions for the micellar solutions of the ethoxylated polysorbate nonionic surfactant, with a stearic alkyl chain and degrees of ethoxylation varying from twenty to fifty. Measurements were made using small angle neutron scattering, SANS, and zeta potential measurements using phase analysis light scattering.

\section{EXPERIMENTAL DETAILS}

Structural measurements and zeta potential measurements were made on ethoxylated polysorbate surfactant solutions in deuterium oxide, $\mathrm{D}_{2} \mathrm{O}$. The structural measurements were made by small angle neutron scattering, SANS, and the zeta potential measurements used phase analysis light scattering.

\section{Small angle neutron scattering.}

The SANS measurements were made using the LOQ (29) and SANS2D (30) diffractometers at the ISIS neutron source, using the white beam time of flight method. On LOQ a wave vector transfer, $\mathrm{Q}$, range of 0.008 to $0.25 \AA^{-1}$ (where $\mathrm{Q}$ is defined as $\mathrm{Q}=(4 \pi / \lambda) \sin (\theta / 2), \lambda$ is the neutron wavelength and $\theta$ is the scattering angle) was covered using a neutron wavelength range of 2 to $10 \AA$ and a sample to detector distance of $4.15 \mathrm{~m}$. The Q range of the measurements on SANS2D was from 0.01 to $0.35 \AA^{-1}$, covered using neutron wavelengths from 2 to $12 \AA$ and a sample to detector distance of $2.42 \mathrm{~m}$. An $8 \mathrm{~mm}$ diameter beam was used with $1 \mathrm{~mm}$ path length samples, contained in quartz spectrophotometer cells, and with measurement times of 20 to 30 mins. The scattering from the cell and solvent were subtracted from the data, and the data were corrected 
for detector response and spectral distribution of the incident beam to establish the scattered intensity, $\mathrm{I}(\mathrm{Q})$, (in $\left.\mathrm{cm}^{-1}\right)$, on an absolute cross-section using standard procedures (31).

\section{Light Scattering.}

Laser Doppler Electrophoresis was used to determine the micelle zeta potential, using a hybrid Malvern Zetasizer Nano, optimised for weakly scattering systems. The instrument uses phase analysis of the light in the cross-over region of the light beam to measure the phase shift arising from the motion of the particle, This can be related to the electrophoretic mobility of the particles and hence their surface charge or potential, as described in detail elsewhere (32).

\section{Materials.}

A range of differently ethoxylated surfactants were synthesised. The ethoxylated sorbitan alkanoate (polysorbate) surfactants were prepared by reacting ethylene oxide with the sorbitan Span60 (octadecanoate), as described elsewhere $(11,33)$. The main focus in the paper is on the poly (ethylene oxide) sorbitan monostearate surfactants which were prepared with 20, 30 and 50 deuterated ethylene oxide groups. The surfactants synthesised are abbreviated as $\mathrm{PES}_{\mathrm{n}} 60$, where $\mathrm{n}$ is 20,30 , and 50. A wider range of $\mathrm{PES}_{\mathrm{n}} 60$ surfactants, with $\mathrm{n}$ ranging from 3 to 50, were previously synthesised (11) and were also measured here. The number of ethylene oxide groups, $\mathrm{n}$, in the commercially available Tween surfactants is 20. Deuterium oxide (Sigma Aldrich) was used to prepare all the aqueous solutions. All the glassware and the quartz spectrophotometer cells were cleaned thoroughly in alkali detergent (Decon90) and rinsed thoroughly in high purity water (Elga Ultrapure, with a conductivity of $10 \mathrm{M} \Omega \mathrm{cm}^{-1}$ ).

\section{Measurements made.}

SANS measurements were made for $\mathrm{PES}_{50} 60$ in $\mathrm{D}_{2} \mathrm{O}$ at surfactant concentrations of 5, 10, 25, and $50 \mathrm{mM}$. At a surfactant concentration of $50 \mathrm{mM}$ measurements were also made for $\mathrm{PES}_{20} 60$ and $\mathrm{PES}_{30} 60$. Measurements were also made for 25 and $50 \mathrm{mM} \mathrm{PES} 5060$ with the addition of 0.1 $\mathrm{M} \mathrm{NaCl}$. Additional measurements were made at a surfactant concentration of $50 \mathrm{mM}$ for $\mathrm{PES}_{\mathrm{n}} 60$, with $\mathrm{n}=3,6,7,9,12,13,17,20,30$, and 50. Light scattering measurements were made for $\mathrm{PES}_{\mathrm{n}} 60$, with $\mathrm{n}=20,30$ and 50 , at a surfactant concentration of $0.5 \mathrm{mM}$, and one other at a lower concentration of $0.05 \mathrm{mM}$. All the measurements were made at a temperature of $25^{\circ} \mathrm{C}$ 


\section{RESULTS and DISCUSSION}

\section{(i) SANS measurements}

The self-assembled structure of the ethoxylated polysorbate surfactants depends upon the alkyl chain length and the degree of ethoxylation, as previously described (11). The SANS data in figure 1 shows the evolution in the structure of the $\mathrm{PES}_{\mathrm{n}} 60$ surfactant, for degrees of ethoxylation, $\mathrm{n}$, from 3 to 50 . The form of the scattering for degrees of ethoxylation from 20 to 50 is consistent with globular interacting micelles. At lower degrees of ethoxylation, from 3 to 9 , the scattering has a $\mathrm{Q}^{-2}$ dependence and has interference features in the form of interference fringes or broad weak Bragg peaks at a $\mathrm{Q}$ value $\sim 0.04$ and $0.08 \AA^{-1}$. This form of the scattering is consistent with lamellar or multi-lamellar structures, in the form of multi-lamellar vesicles or a lamellar phase. The $\mathrm{Q}$ value of the Bragg peaks equate to a lamellar spacing $\sim 50 \AA$. The scattering for $\mathrm{n}$ in the range 12 to 17 is intermediate between the two extremes, and in the form of micellar / lamellar coexistence. This evolution in structure is broadly consistent with the data previously reported for $\mathrm{PES}_{\mathrm{n}} 20$ (lauric alkyl chain) and $\mathrm{PES}_{\mathrm{n}} 60$ (11).

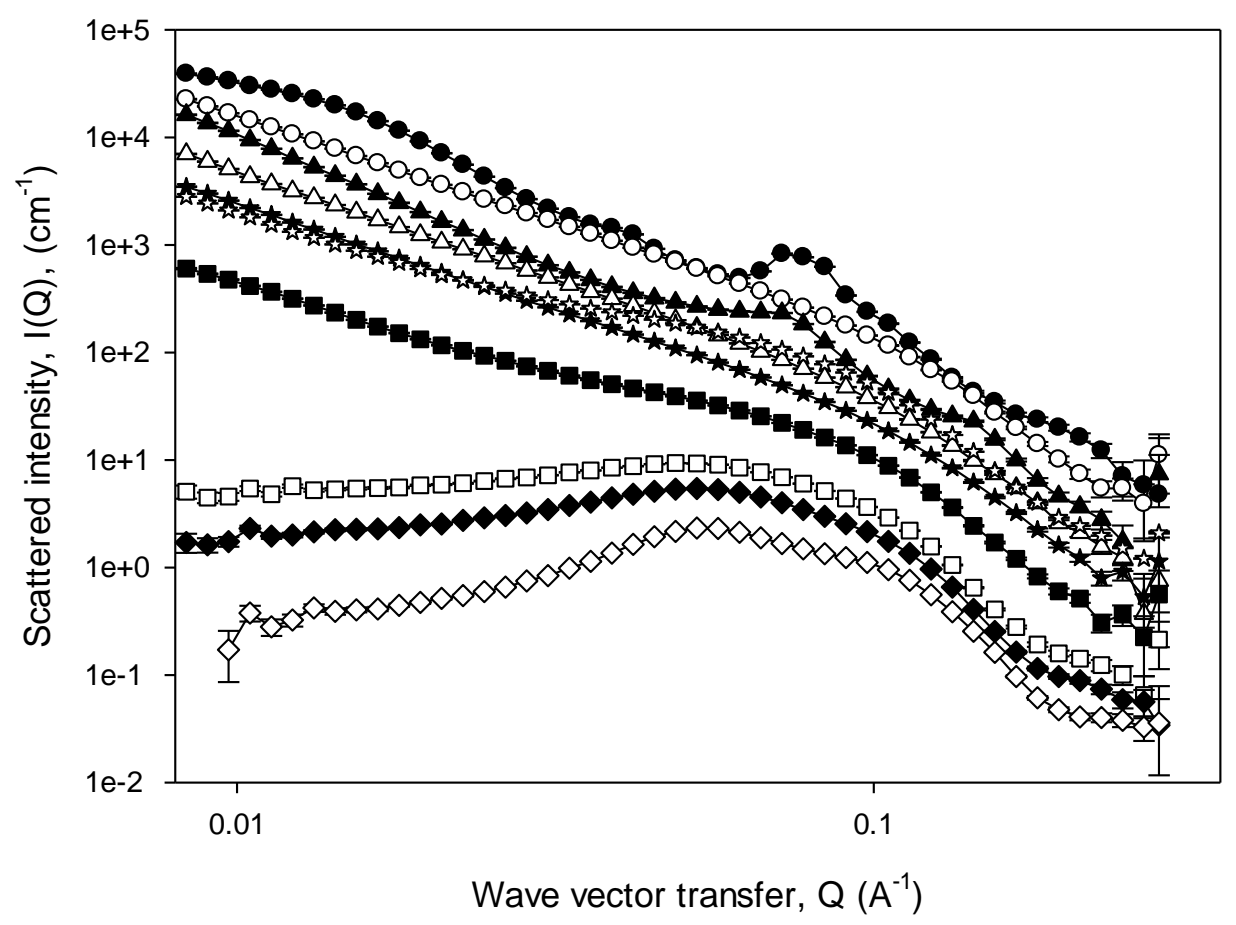

FIGURE 1. SANS data for $50 \mathrm{mM} \mathrm{PES} 60$ in $D_{2} O$, for $n=50(\diamond), 30(\diamond), 20(\square), 17(\boldsymbol{\square})$, 13( $*), 12(\star), 9(\Delta), 7(\Delta), 6(O)$, and $3(\bullet)$, from bottom to top curve, and each curve is shifted vertically by $x 2$. 
The SANS data for the degrees of ethoxylation of 20,30 and 50 in figure 1 are analysed quantitatively using a model of interacting core-shell globular micelles. The scattering from interacting globular micelles, within the decoupling approximation, can be written as (34),

$I(Q)=N_{p}\left[S(Q)\left|\langle F(Q)\rangle_{Q}\right|^{2}+\left\langle|F(Q)|^{2}\right\rangle_{Q}-\left|\langle F(Q)\rangle_{Q}\right|^{2}\right]$

$\mathrm{S}(\mathrm{Q})$ is the inter-micellar structure factor, $\mathrm{N}$ is the micelle number density, and $\mathrm{F}(\mathrm{Q})$ the micelle form factor. $\mathrm{S}(\mathrm{Q})$ is modelled using the rescaled mean spherical approximation, RMSA, approach (35), where the inter-micellar interaction is modelled as a hard-sphere + Yukawa screened Coulombic potential; and the Yukawa potential represents a soft tail to the core hardsphere potential. $\mathrm{S}(\mathrm{Q})$ is defined by a surface charge, $\mathrm{z}$, or surface potential, the number density, $\mathrm{N}$, the micelle diameter, $\sigma$, and the Debye-Huckel inverse screening length, $\kappa$. In the absence of charge or for a low charge this reduces to the Percus-Yevick, PY, hard-sphere S(Q) (36). The form factor, $F(Q)$, which describes the micelle size, shape and internal structure, is represented by the familiar core-shell form factor (37). The micelle structure is hence modelled as an inner core of radius R1 containing the alkyl chains, and an outer shell of radius R2 $(\sigma=2 R 2)$ containing the headgroups and associated hydration (34). Molecular constraints are included, such that the inner radius is limited to the fully extended chain length of the surfactant, lc, for spherical micelles. The outer radius, $\mathrm{R} 2$, is an adjustable parameter in the model, to accommodate variations in the packing constraints of the ethoxylated headgroups from space filling requirements. From known molecular volumes, dimensions and neutron scattering length (see table $\mathrm{S} 1$ in the Supporting Information) the scattering can be calculated on an absolute scale using equation 1 and the standard form of $\mathrm{F}(\mathrm{Q})$ for a core-shell structure (see equation $\mathrm{S} 1$ in the Supporting Information). The calculated $\mathrm{I}(\mathrm{Q})$ is compared with the SANS data using a least squares criterion; with adjustable model parameters of $v, \mathrm{R} 2$, and $\mathrm{z}$. The modelling here is relatively insensitive to varying $\kappa$ due to the low surface charge values and the impact of electrolyte (see later), and a fixed value of $0.01 \AA^{-1}$ was used. An acceptable model fit requires the functional form of the data to be represented and the absolute scattered intensity to be within $\pm 20 \%$ of the data.

The SANS data for $50 \mathrm{mM} \mathrm{PES}$ 60, for $\mathrm{n}=20,30$ and 50, are shown in figure 2 along with the corresponding model fits. The key model parameters are summarised in table 1 . 


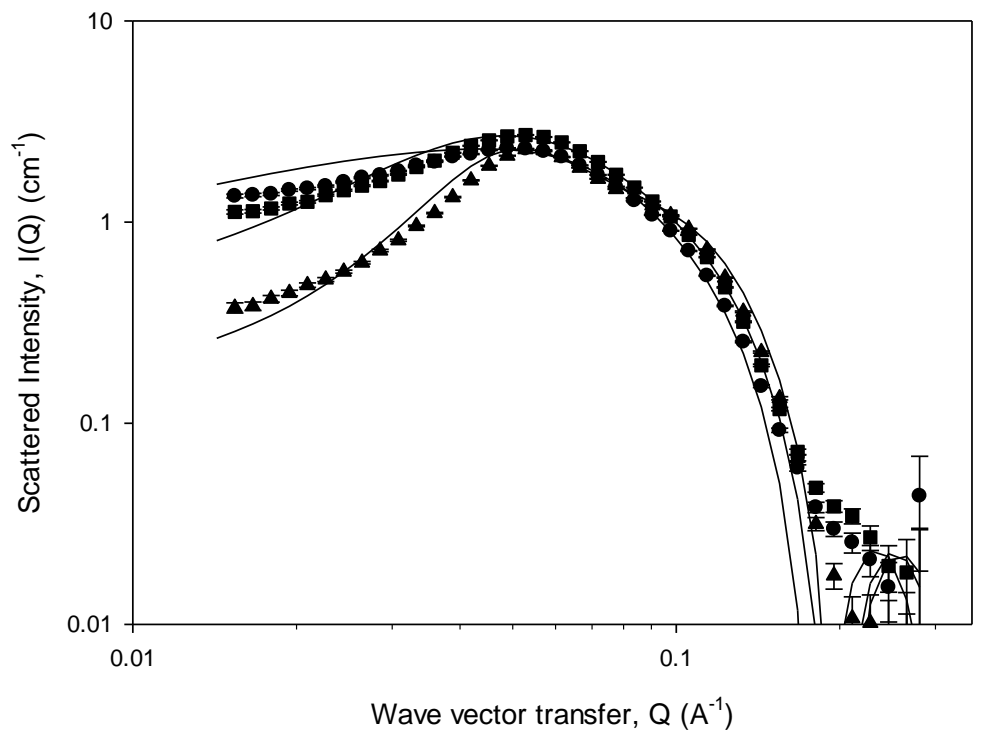

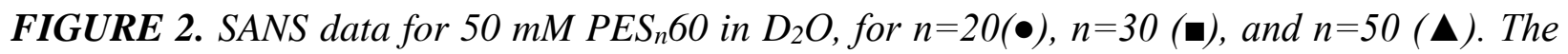
solid lines are model fits for the key model parameters in table 1 .

TABLE 1. Key model parameters for core-shell model fits to SANS data for $\mathrm{PES}_{n} 60$ in $\mathrm{D}_{2} \mathrm{O}$

(a) $P E S_{n} 60$ at $50 \mathrm{mM}$ for $n$ from 20 to 50

\begin{tabular}{|c|c|c|c|c|c|}
\hline $\begin{array}{c}\begin{array}{c}\text { Degree of } \\
\text { ethoxylation, } \\
n\end{array} \\
\end{array}$ & $\begin{array}{c}\text { Aggregation } \\
\text { number, } v \\
( \pm 5)\end{array}$ & $\begin{array}{c}\text { Surface } \\
\text { charge, } z \\
( \pm 1)\end{array}$ & $\begin{array}{c}\text { Degree of } \\
\text { ionisation, } \boldsymbol{\delta}, \\
(\mathbf{\pm 0 . 0 1 )} \\
\end{array}$ & $\mathrm{R} 1( \pm 1 \AA ̊)$ & $\mathrm{R} 2( \pm 1 \AA)$ \\
\hline 50 & 100 & 12 & 0.12 & 22 & 45 \\
\hline 30 & 97 & 7 & 0.07 & 22 & 40 \\
\hline 20 & 90 & 3 & 0.03 & 22 & 37 \\
\hline
\end{tabular}

(b) $P E S_{50} 60$ at concentrations from 5 to $50 \mathrm{mM}$

\begin{tabular}{|c|c|c|c|c|c|}
\hline $\begin{array}{c}\text { Surfactant } \\
\text { concentratio } \\
\text { n }(\mathbf{m M})\end{array}$ & $\begin{array}{c}\text { Aggregation } \\
\text { number, } v \\
( \pm 5)\end{array}$ & $\begin{array}{c}\text { Surface } \\
\text { charge, } z \\
( \pm 1)\end{array}$ & $\begin{array}{c}\text { Degree of } \\
\text { ionisation, } \boldsymbol{\delta} \\
(\mathbf{\pm 0 . 0 1 )}\end{array}$ & R1 $( \pm 1 \AA)$ & $\mathrm{R} 2( \pm 1 \AA)$ \\
\hline 50 & 100 & 12 & 0.12 & 22 & 45 \\
\hline 25 & 94 & 9 & 0.07 & 22 & 45 \\
\hline 10 & 72 & 9 & 0.13 & 21 & 43 \\
\hline 5 & 79 & 9 & 0.11 & 20 & 43 \\
\hline
\end{tabular}


In the data presented the micelle aggregation number varies from $\sim 90$ to 100 , depending upon the degree of ethoxylation; and the values summarised in table 1a are consistent with previously reported data (11). Furthermore for this range of aggregation numbers the micelles remain spherical, with an inner core radius of $22 \AA$ (corresponding to the fully extended stearic alkyl chain, lc), and an outer radius which increases from 37 to $45 \AA$ as the degree of ethoxylation increases. The hydration of the outer shell corresponds to a number of water molecules / EO that varies from $\sim 2$ to $\sim 5$ as the degree of ethoxylation increases from 20 to 50, and are within the range of physically expected values. The contribution from $S(Q)$, which affects the data predominantly at lower $\mathrm{Q}$ values, $\mathrm{Q}$ values $\leq 0.05 \AA^{-1}$, is not accounted for by a purely hardsphere structure factor as encountered in previous nonionic surfactant micelles (13-17, 19, 20, 24-26). An $S(Q)$ derived from the soft -sphere Yukawa potential, with an effective surface charge which increases as the degree of ethoxylation increases, is used to describe the data. We will return to this point later in the discussion.

Figure 3 shows the SANS data for PES 5060 at surfactant concentrations of 5, 10, 25, and $50 \mathrm{mM}$, and the associated model fits. The key model parameters are summarised in table $1 \mathrm{~b}$.

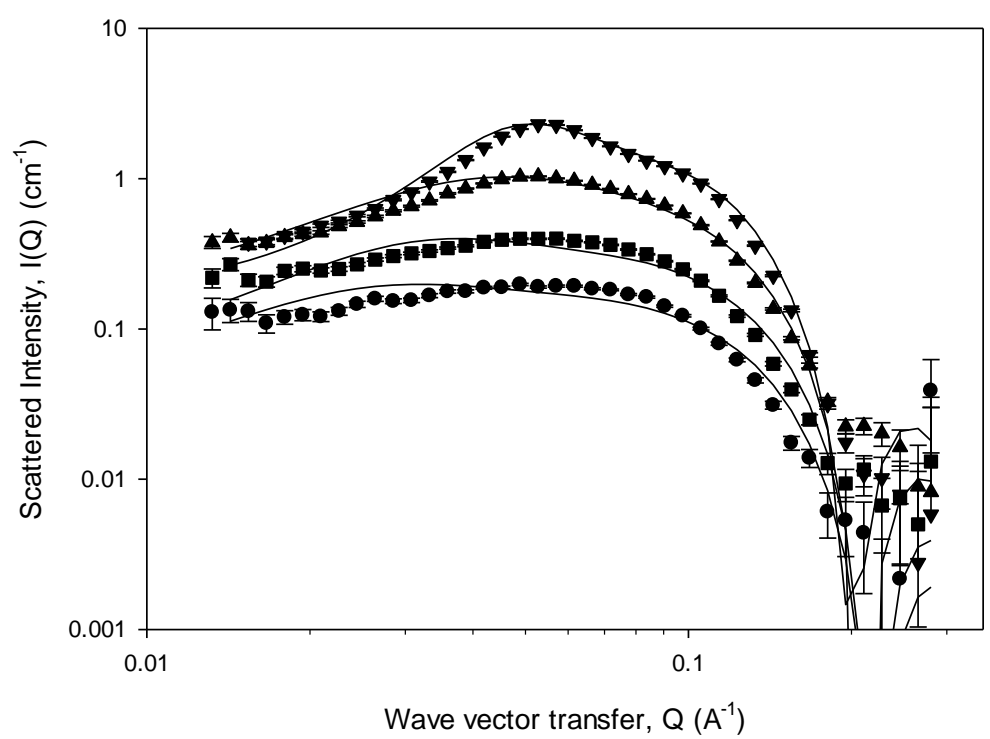

FIGURE 3. SANS data for PES 5060 in $\mathrm{D}_{2} \mathrm{O}$ at surfactant concentrations of $5 \mathrm{mM}(\bullet), 10 \mathrm{mM}$ (-), $25 \mathrm{mM}(\mathbf{\Delta})$, and $50 \mathrm{mM}(\mathbf{\nabla})$. The solid lines are model fits for the key model parameters in table $1 b$. 
TABLE 2. Variation in surface charge for $P E S_{n} 60$, from analysis of SANS data and zeta potential measurements

\begin{tabular}{|c|c|c|c|c|c|c|}
\hline $\begin{array}{c}\text { Surfactant } \\
\text { concentration } \\
(\mathbf{m M})\end{array}$ & $\begin{array}{c}\text { Degree of } \\
\text { ethoxylation, } \\
\text { n }\end{array}$ & $\begin{array}{c}\text { Aggregation } \\
\text { number, } v \\
\quad( \pm 5)\end{array}$ & $\begin{array}{c}\text { Surface } \\
\text { charge, } \mathrm{z}, \\
\quad( \pm 1)\end{array}$ & $\begin{array}{c}\text { Degree of } \\
\text { ionisation, } \\
\delta,( \pm 0.01)\end{array}$ & $\begin{array}{c}\text { Charge / } \\
\text { EO } \\
( \pm 0.2 \times 10 \\
3)\end{array}$ & $\begin{array}{c}\text { Surface } \\
\text { charge } \\
\text { density } \\
( \pm 0.8 \mathrm{mC} \\
\left.\mathbf{m}^{-2}\right)\end{array}$ \\
\hline 50 & 50 & 100 & 12 & 0.12 & -2.4 & -7.6 \\
\hline 25 & 50 & 94 & 9 & 0.07 & -1.4 & -4.5 \\
\hline 10 & 50 & 72 & 9 & 0.13 & -2.6 & -6.2 \\
\hline 5 & 50 & 79 & 9 & 0.11 & -2.2 & -5.3 \\
\hline 50 & 30 & 97 & 7 & 0.07 & -2.3 & -5.6 \\
\hline 50 & 20 & 90 & 3 & 0.03 & -1.5 & -2.7 \\
\hline
\end{tabular}

At all the concentrations measured the $\mathrm{PES}_{50} 60$ micelles are spherical, and the aggregation number increases from $\sim 70$ to $\sim 100$ as the surfactant concentration increases from 5 to $50 \mathrm{mM}$. The inner radius, $\mathrm{R} 1$, has an average value $\sim 21 \AA$, and the outer radius, $\mathrm{R} 2$, has an average value $44 \AA$; and both dimensions increase slightly as the surfactant concentration and aggregation number increases. However, the notable feature of the data analysis is that, along with the data in figure 2, the structure factor, $S(Q)$, is not consistent with a purely hard-sphere potential. The structure factor has been modelled as a soft-sphere screened Coulombic Yukawa potential with an effective surface charge varying from 7 to 12 . Expressed as a ratio to the aggregation number, that is an effective degree of ionisation, $\delta$, it is roughly constant with surfactant concentration with an average value $\sim 0.11$. This is consistent with the data in table 1 a for the variation in $\delta$ with the degree of ethoxylation at a fixed surfactant concentration.

The significance of the form of $S(Q)$ and the inadequacy of the hard-sphere $S(Q)$ at these relatively low volume fractions is illustrated in figure 4. 


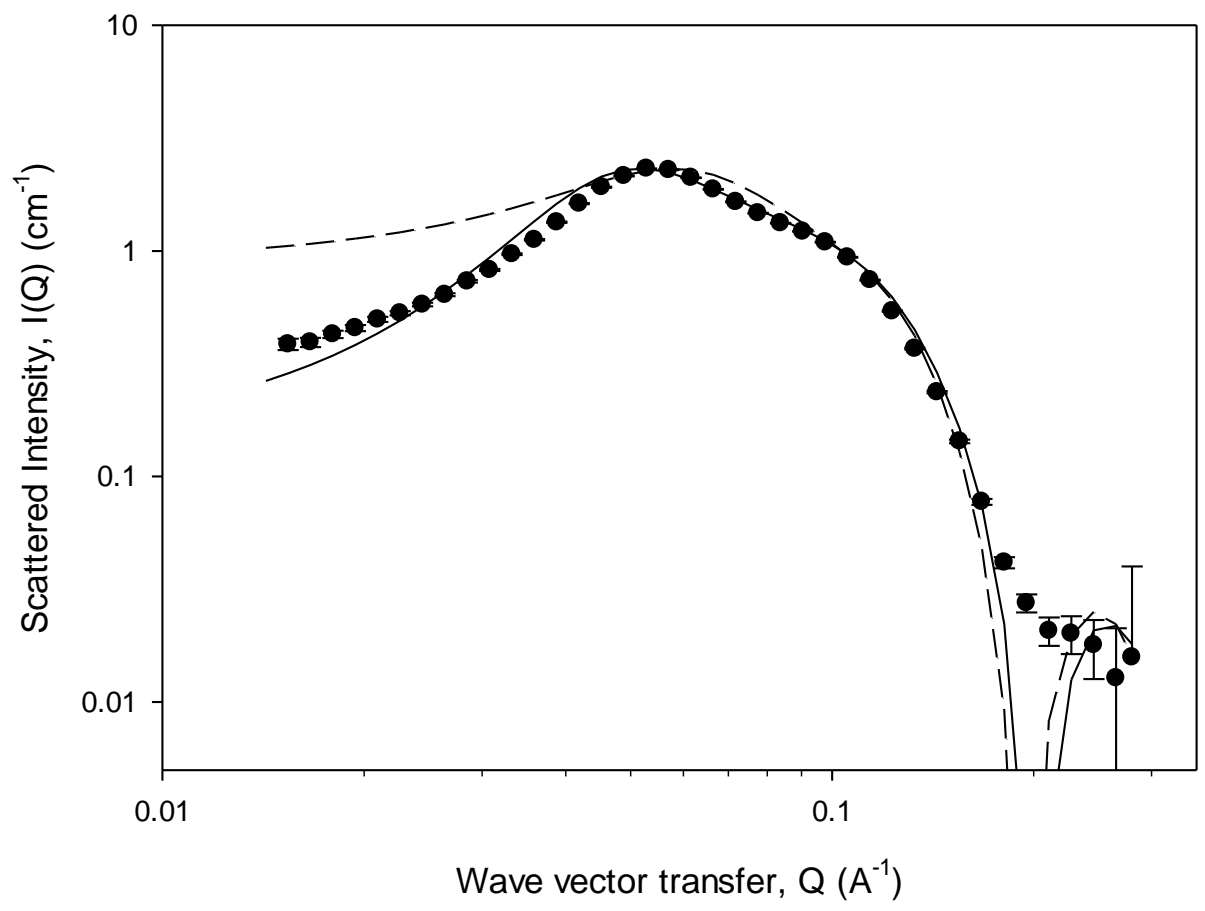

FIGURE 4. SANS data for $50 \mathrm{mM} P E S_{50} 60$ in $\mathrm{D}_{2} \mathrm{O}$. The solid line is a model fit using the key model parameters in table $1 b$. The dashed line is a model calculation using a hard-sphere $S(Q)$ only.

Figure 4 shows the SANS data for $50 \mathrm{mM}$ PES $_{50} 60$ with the model fit for the parameters summarised in table 1 shown as a solid line. The dashed line shows the effect of including $S(Q)$ as only a hard-sphere $S(Q)$ without any effective charge on the micelles. The inclusion of only the excluded volume hard-sphere $S(Q)$ substantially underestimates the contribution of $S(Q)$ to the data.

In table 2 the effective charge for the different model fits are summarised, and also expressed as a degree of ionisation, charge / ethylene oxide group, and a surface charge in $\mathrm{mCm}^{-2}$. The variation in the surface charge on the micelles with the degree of ethoxylation varies approximately linearly, as shown in figure 5 


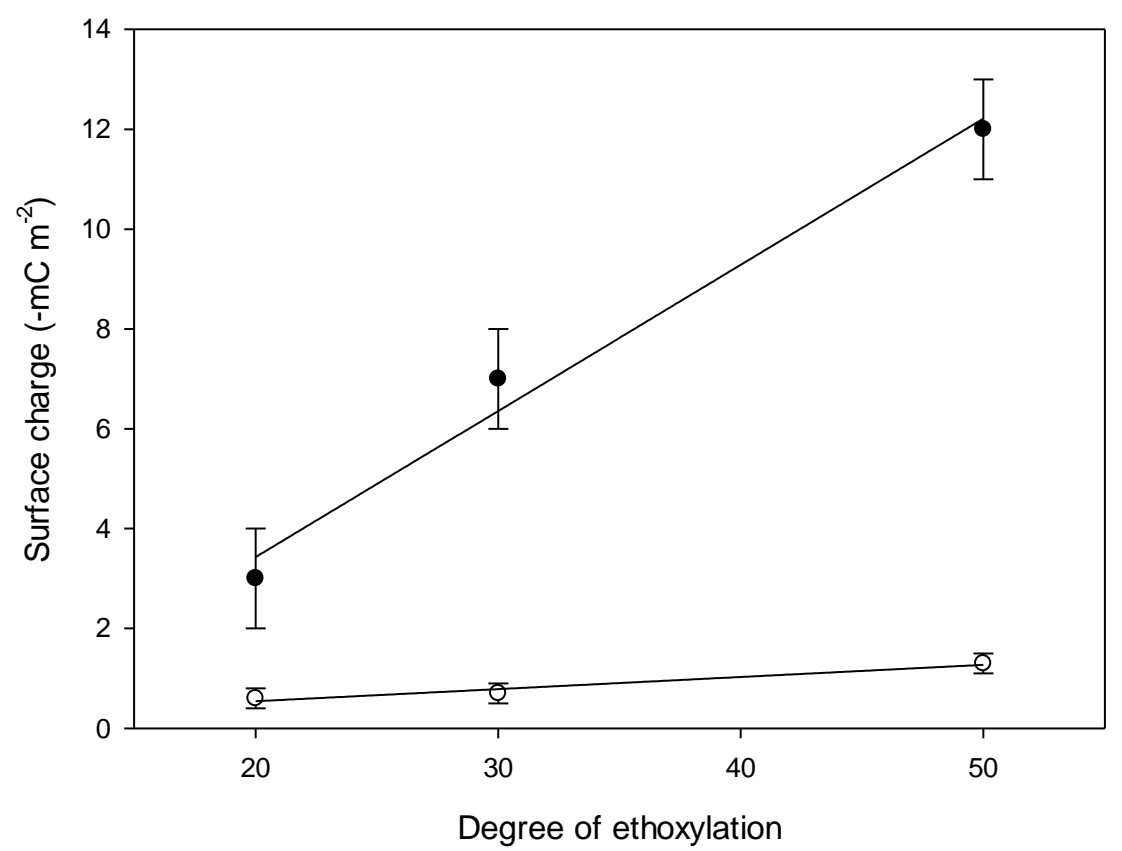

FIGURE 5. Variation in micelle surface charge with degree of ethoxylation for $P E S_{n} 60$, for the SANS data from table $2(\bullet)$ and from the zeta potential measurements in table $3(O)$.

\section{(ii) Zeta potential measurements}

Laser Doppler electrophoresis was also used to determine the zeta potential of the micelles, as described earlier and in more detail elsewhere (32). The phase shift of the Moire fringe during electrophoretic perturbation of the colloidal particles is directly related to the electrophoretic mobility of the particles, and hence the surface charge or potential, can be easily determined (38).

At the concentrations used for the SANS measurements $(\geq 5 \mathrm{mM})$ the strong repulsive $\mathrm{S}(\mathrm{Q})$ inter-particle interaction restricts the motion of the particles under electrophoretic conditions and results in artificially low mobilities. This is illustrated in figure S1 in the Supporting Information, where the repulsive $S(Q)$ introduces a long wavelength sinusoidal variation in the phase plot. The addition of electrolyte, as also illustrated in the SANS measurements in figure 6, does not suppress the contribution of $\mathrm{S}(\mathrm{Q})$. Hence a stock $5 \mathrm{mM}$ solution was diluted whilst viewing the electrophoretic phase plot under both fast and slow field reversal conditions. Dilution to $0.5 \mathrm{mM}$ was found to be sufficient to minimise the effects of the repulsive interactions, as illustrated by the phase plot in figure S2 in the Supporting Information. Under these dilute conditions and for relatively small micelles extended measurement times were 
required. The data were obtained using a sequence of six measurements with $30 \mathrm{sec}$ pauses between runs to minimise the effects of Joule heating, and typical data are shown in figure S3 in the Supporting Information. The zeta potential and surface charge measurements are summarised in table 3.

Table 3. Surface charge and zeta potential data for $P E S_{n} 60$, for $n=20,30$ and 50 micelles at a concentration of $0.5 \mathrm{mM}$ from LDE measurements.

\begin{tabular}{|c|c|c|}
\hline $\begin{array}{c}\text { Surfactant degree of } \\
\text { ethoxylation, } \mathbf{n}\end{array}$ & Zeta potential $(\mathbf{m V})$ & Surface charge $\left(\mathbf{m C ~}^{-\mathbf{2}}\right)$ \\
\hline 20 & $-8( \pm 2)$ & $-0.6( \pm 0.2)$ \\
\hline 30 & $-9( \pm 2)$ & $-0.7( \pm 0.2)$ \\
\hline 50 & $-18( \pm 1)$ & $-1.3( \pm 0.1)$ \\
\hline
\end{tabular}

The zeta potential measurements are in broad qualitative agreement with the interpretation of the SANS data. That is, there is a finite surface charge evident on the micelles which increases with increasing degree of ethoxylation. However quantitatively the zeta potential measurements give values that are systematically lower than those inferred from the SANS data. The zeta potential measurements were made at much lower surfactant concentrations than the SANS measurements, $\sim 10$ to 100 times lower. It is not clear what impact this has on the micelle structure and surface charge, as there is insufficient signal to make the SANS measurements at an equivalent low concentration. However it is clear that there was a sufficient solution structure factor manifested in the phase plot of the zeta potential measurement at the higher concentrations $(5 \mathrm{mM})$ which necessitated dilution by 10 fold or more to allow the surfactant micelles to become "free moving" over the gauge volume of the Mach-Zehnder, M-Z, interferometric crossover. This itself corroborates the inclusion of the structure factor in the SANS data treatment.

\section{(iii) Discussion}

For degrees of ethoxylation of 20 and less the SANS data can be adequately described by the hard core $\mathrm{S}(\mathrm{Q})$, and within error the value of $\delta$ is relatively close to zero. At the higher degrees of ethoxylation the additional interaction associated with an effective surface charge is 
increasingly evident. The partial charge on the ether oxygen group has been previously implicated in the interaction of poly(ethylene glycol) with ionic surfactants (27) and the adsorption of poly(ethylene oxide) onto mica (28). It is postulated here that for the high degrees of ethoxylation the partial charge on the ether oxygen is sufficient to manifest itself as an effective charge on the micelle. It has not been observed in Triton X-100 and Brij35 nonionic surfactants $(22,23)$ which have degrees of ethoxylation of 10 and 23 respectively. It has also not been generally reported for $\mathrm{C}_{12} \mathrm{E}_{8}$ or $\mathrm{C}_{12} \mathrm{E}_{12}$ nonionic surfactants $(19,20)$; although it was inferred in one SANS study on $\mathrm{C}_{12} \mathrm{E}_{8}$ (18). It has not been observed in the pluronic block copolymer micelles, where the degree of ethoxylation is much higher, and ranges from 25 to 100 (24-26). In the analysis of the pluronic micelle SANS data the inter-micellar interactions are incorporated using the P-Y hard-sphere $\mathrm{S}(\mathrm{Q})$. However the hard-sphere diameter is used as a fitting parameter $(25,26)$ and this may partially compensate for or mask any charge effects.

It seems that although the appearance of an effective surface charge depends upon the degree of ethoxylation being above a threshold value, on the basis of the data from a wider range of nonionic systems it is not alone a sufficient requirement. The differences between the ethoxylated polysorbate surfactants and particularly the pluronic block copolymers implies that the structure and distribution of the poly(ethylene oxide) groups is important. The more disordered and less compact structure of the pluronic block copolymers results in no equivalent measurable effect, and this implies either a screening of the partial charges or a more disordered and spatially extended distribution. Viera et al (33) showed that in the adsorption at the air-water interface of the pluronics $\mathrm{EO}_{23} \mathrm{PO}_{52} \mathrm{EO}_{23}$ and $\mathrm{EO}_{9} \mathrm{PO}_{22} \mathrm{EO}_{9}$ there was disorder resulting in some intermixing between the ethylene oxide and propylene oxide groups. It was demonstrated that this interpretation was consistent with a greater degree of intermixing in the associated micellar structures than was assumed in the core-shell model reported for pluronic micelles (39). The less hydrophilic environment encountered there for the ethylene oxide groups would have an effect on any net partial charge that might be evident. The observation of the effect could also depend upon the micelle concentration or volume fraction as well as the degree of ethoxylation.

The scattering studies on non-ionic surfactant micelles have been generally measured at relatively high surfactant concentrations (13-23). This implies that if the degree of ethoxylation and the surfactant concentration are sufficiently low then the effect will not be observed. On the other hand if the surfactant concentration is too large $\mathrm{S}(\mathrm{Q})$ could well be dominated by the hard- 
sphere term. Hence it is postulated that to observe the effect there needs to be a sufficiently high degree of ethoxylation and the micelle concentration or volume fraction should be intermediate so that the inter-micellar interactions are sensitive to the soft tail of the potential.

However the impact of the coherent summation effect of a series of partial charges is different to that of dissociated charges in an ionic surfactant micelle. The clearest demonstration of this is shown in figure 6.

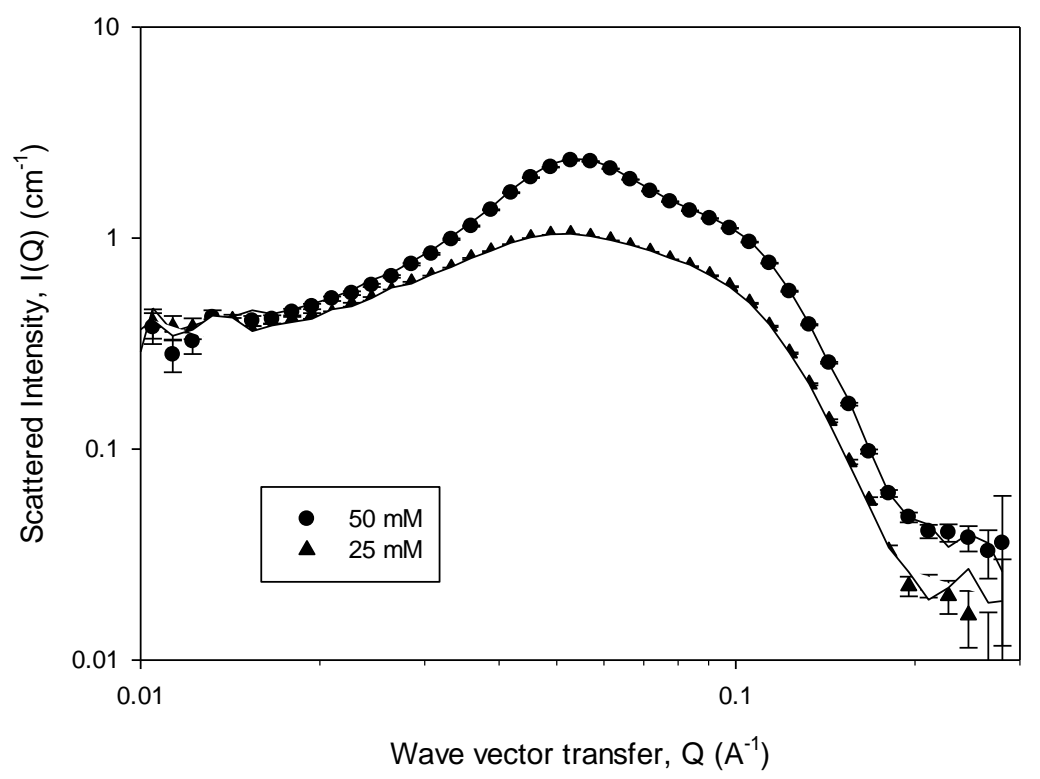

FIGURE 6. SANS data for PES $\mathrm{P}_{50} 60$ in $\mathrm{D}_{2} \mathrm{O}$ at $25 \mathrm{mM}(\mathbf{\Delta})$ and $50 \mathrm{mM}(\bullet)$ in the absence of electrolyte. The solid lines are the data with the addition of $0.1 \mathrm{M} \mathrm{NaCl}$.

Figure 6 shows the SANS data for $\mathrm{PES}_{50} 60$ at surfactant concentrations of 25 and $50 \mathrm{mM}$, in the absence and presence of $0.1 \mathrm{M} \mathrm{NaCl}$. The addition of electrolyte has no impact upon the scattering, as might be expected for a non-ionic surfactant micelle. For ionic surfactants the addition of electrolyte screens the charge and reduces the inverse Debye-Huckel screening length, from $\geq 30$ to $<10 \AA$. This generally has the effect of substantially reducing the suppression of the scattering intensity at low $Q$ due to the $S(Q)$ contribution as the extent of the coulombic contribution to the potential is reduced $(34,40)$. The invariance with the addition of electrolyte is a further indication of the more distributed nature of the effective surface charge of the ethoxylated polysorbate surfactants. An insensitivity to electrolyte has been observed in other charged systems; for example, the swelling of polyelectrolyte brushes $(41,42)$. In the 'osmotic' regime the extent of polyelectrolyte brushes is insensitive to added electrolyte and are 
stretched simply as a result of the osmotic pressure exerted by the counterions trapped inside the brush. At higher electrolyte concentrations, when the added electrolyte concentration exceeds the concentration of free counterions in the brush, the 'salted brush' regime, the swelling depends on the electrolyte concentration. A similar impenetrability of the added electrolyte into the ethoxylated headgroup region of the ethoxylated polysorbate surfactant micelles, as encountered in the 'osmotic' regime of polyelectrolyte brushes, would minimise any screening effects on the distributed nature of the partial charges arising from the ethylene oxide groups.

Possible alternative sources of the origin of the surface charge or other forms of repulsive interaction have been considered. The sorbitan alkanoate (sorbitan Span60), which is the precursor surfactant prior to ethoxylation, is uncharged. Hence the origin of the charge on the micelles cannot be attributed to any fraction of sorbitan that is not ethoxylated, and in any case the stoichiometry of the reaction implies that the fraction is small. Furthermore the method of synthesis and subsequent characterisation $(11,33)$ would imply that it in any case a small fraction. In particular, the potassium t-butoxide catalyst used in the reaction of the Span with the ethylene oxide is neutralised by $\mathrm{HCl}$. The resulting mixture is freeze dried to remove water and residual $\mathrm{HCl}$. The residue is dissolved in ethyl acetate followed by rotary evapouration to remove residual $\mathrm{KCl}$, and it is unlikely that there is a measurable amount of electrolyte remaining after this stage of extraction and purification. removal of other adventitious ions is relatively difficult (45) and the usual neutral concentrations of $\mathrm{H}^{+}$and $\mathrm{OH}^{-}$may be present In the measurement of the forces between fluid interfaces stabilised by nonionic surfactants others $(43,44)$ have reported a charge contribution. This has been attributed mainly to the adsorption of hydroxyl $\left(\mathrm{OH}^{-}\right)$ions at the interface. Bergeron et al (44) showed how increasing the surfactant concentration displaces the surface ions, and so the surface charge was influenced by surfactant concentration, $\mathrm{pH}$ and ionic strength. This is directly applicable to the oil-water and air-water interfaces, but not the micelle surface. Earlier in the analysis and discussion the possibility of a purely hard-sphere $\mathrm{S}(\mathrm{Q})$ at the volume fractions and structures determined was eliminated. If some of the EO chains were attached to the sorbitan groups as long chains then an effective longer ranged repulsion could occur The structural determination of these surfactants at the airwater and oil-water interface $(11,12)$, where a relatively compact layer with a uniform distribution of EO chains is observed, imply that this is unlikely. It was previously shown (11) at the air-water interface that the area/molecule for the Tween-like molecules with EO content ranging from 3 to 50 (that is, with an average of 1 to 16 EO's per chain) accurately followed a 
linear dependence. Any significant polydispersity in the ethoxylation or distribution of the EO chains would give rise to strong deviation from that linearity. For example the variation in the area / molecule for the liner poly(ethylene glycol) ether deviates from a liner dependence for greater than $\mathrm{EO}_{5}$ due to the chains adopting a Gaussian form (46). This further mitigates against a long ranged steric repulsion from the EO chains

The surface charge derived from the SANS and zeta potential measurements are in broad qualitative agreement. That is, the micelles have a finite surface charge greater than that expected for nonionic surfactants, and the surface charge increases as the degree of ethoxylation of the surfactant headgroup increases. However the quantitative agreement is less good. The surface charge values determined from the SANS measurements for ionic surfactants are generally in good agreement with the 'dressed micelle' model $(39,47)$. The 'dressed micelle' model is derived from a solution of the non-linear Poisson-Boltzmann equation, which describes the ionic distribution about the micelle which is assumed to depend upon the radial distance only. It determines a finite bound fraction of counterions and does not require the concept of the double layer or Stern layer to be specifically included. As such it can be considered as the charge or potential at the surface of the hydrated headgroup region of the micelle. The zeta potential is defined as the potential in the interfacial double layer at the location of the 'slipping plane'. As such it is not generally equal to the potential at the Stern layer or the surface layer of the double layer (48). Hence it is argued here that the potential and charge values obtained from the zeta potential measurements will be systematically lower than those obtained from the SANS analysis, although no quantification of the difference has been calculated or systematically reported. This is coupled with the uncertainties arising from the differences in solution concentration that the two measurements were made at, and described earlier. The zeta potential measurements were made at a solution concentration of $0.5 \mathrm{mM}, 10$ to 100 times lower that the concentrations used for the SANS studies. The extent to which the micelle structure, size and charge depends on surfactant concentration in the concentration range of 0.5 to $5 \mathrm{mM}$ is uncertain and not generally measurable by SANS.

\section{SUMMARY}

SANS measurements have been used to study the self-assembly of a range of ethoxylated polysorbate surfactants. For high degrees of ethoxylation globular interacting micelles are formed. Unlike that normally encountered with nonionic surfactants the inter-micellar 
interactions are not explained by the hard-sphere excluded volume $S(Q)$. An effective surface charge, which varies with the degree of ethoxylation, is required. This is assigned to a coherent summation of the partial charges associated with the ether oxygens of the ethylene oxide groups. Zeta potential measurements are broadly consistent with the interpretation of the SANS data, and also indicate a net surface charge on the micelles. The inter-micellar interaction is unaffected by added electrolyte, in contrast to ionic surfactant micelles. These systems raise the interesting prospect of exploiting the charged nature of the micelles in circumstances where electrolyte might normally have a negative influence, such as promoting unwanted micellar growth or insolubility.

\section{ACKNOWLEDGEMENTS}

We acknowledge the use of the diffractometers LOQ and SANS2D at ISIS, the provision of beam time, and the assistance of Ann Terry.

\section{SUPPORTING INFORMATION}

Some further details associated with the modelling of the SANS data are included in the Supporting Information, in the form of equations and tables, and of the zeta potential measurements in the form of figures. The material is available free of charge via the internet at http://pubs.acs.org.

\section{AUTHOR INFORMATION}

Corresponding author: jeff.penfold@stfc.ac.uk

Author Contributions: All the authors have given their approval of the final version of the manuscript.

Funding Sources: The neutron beam time was provided by STFC's ISIS facility. 


\section{REFERENCES}

(1) Nonionic surfactants: Physical Chemistry, Surfactant Science Series, Vol 23, Ed M. J. Schick, Marcel Dekker, NY, 1987

(2) van Os, N. M.; Haak, J. R.; Rupert, L. A. M. Physico-chemical properties of selected anionic, cationic and non-ionic surfactants, Elsevier, Amsterdam, 1993

(3) Shen, L.; Guo, A.; Zhu, X. Tween surfactants: adsorption. Self-organisation and protein resistance, Surface Science, 2011, 105. 494-499

(4) Wan, L. S. C.; Lee, P. F. S. Cmc of polysorbates, J. Pharm. Sci. 1974, 63, 136-137

(5) Kragel, J.; Wustneck, R.; Husband, F.; Wilde, P. J.; Makievski, A. V.; Grigoriev, D. O.; Li, J. B. Properties of mixed protein / surfactant adsorption layers, Coll. Surf. B, 1999, 12, 399-407

(6) Ruiz-Henestrosa, V. P.; Sanchez, C. C.; Patino, J. M. R. Adsorption and foaming characteristics of Soy Globulins and Tween 20 mixed systems, Ind. End. Chem. Res. 2008, 47, 2876-2885

(7) Sharma, S. C.; Warr, G. C. Phase phenomena, self-assembly and emulsification of Tween80 / water mixtures with Limonene and perfluoromethyldecalin, Langmuir, 2012, $28,11707-11713$

(8) Bhattacharjee, J.; Verma, G.; Aswal, V. K.; Dale, A. A.; Nagarsenker, M. S.; Hassan, P. A. Tween 80 - sodium deoxycholate mixed micelles: structural characterisation and application in deoxoruicin delivery, J. Phys. Chem. B, 2010, 114, 16414-16421

(9) O’Sullivan, S. M.; Woods, J. A.; O’Brien, N. M. Use of Tween40 and Tween60 to deliver a mixture of cytochemicals to human colonic adenoracinoma cells, Brit. J. Nutr. 2004, $91,757-764$

(10) Webb, S. D.; Cleland, J. L.; Carpenter, J. F.; Randolph, T. W. A new mechanism for decreasing aggregation of recombinant Human Interferon- $\gamma$ by a surfactant: slow dissolution of lypophilised formulations is a solution containing $0.03 \%$ polysorbate $20, J$. Pharm. Sci. 2007, 91, 543-558 

P.; Terry, A. Adsorption at the air-water and oil-water interfaces and self-assembly in aqueous solution of ethoxylated polysorbate non-ionic surfactants, Langmuir, 2015, 31, 3003-3011

Penfold, J.; Thomas, R. K.; Li, P. X.; Petkov, J. T.; Tucker, I. M.; Cox, A. R.; Hedges, N.; Webster, J . R. P.; Skoda, M. Surface self-assembly in hydrophobinethoxylated polysorbate non-ionic surfactant mixtures: manipulating the structural evolution through variable amounts of surfactant ethoxylation, Langmuir, 2014, 30, 9741-9751

Hayter, J. B.; Zulauf, M. Attractive interactions in critical scattering from nonionic micelles, Coll. Polym. Sci. 1982, 260, 1023-1028

Magid, L. J.; Triolo, R.; Johnson, J. S. Small angle neutron scattering of critical phenomena in aqueous solutions of $\mathrm{C}_{12} \mathrm{E}_{8}$, a nonionic amphiphile, J. Phys. Chem. 1984, 88, 5730-5734

Zulauf, M.; Weckstrőm, H.; Hayter, J. B.; Degiorgio, V.; Corti, M. A neutron scattering study of micelle structure in isotopic aqueous solutions of polyoxyethylene surfactants, J. Phys. Chem. 1985, 89, 3411-3417

(16) Cantu, L.; Corti, M.; Degiorgio, V.; Hoffman, H.; Ulbricht, W. Nonionic micelles in mixed water-glycerol solvent, J. Coll. Int. Sci. 1987, 116, 384-389

Triolo, R.; Magid, L. J.; Johnson, J. S.; Childs, H. R. Small angle scattering from aqueous micellar solutions of a non-ionic surfactant as a function of temperature, J. Phys. Chem. 1982, 88, 3689-3695

(18) Penfold, J.; Staples, E.; Tucker, I.; Cummins, P. The structure of non-ionic micelles in less polar solvents, J. Coll. Int. Sci. 1997, 185, 424-431

$$
\text { Penfold, J.; Thomas, R. K.; Dong, C. C.; Tucker, I.; Metcalfe, K.; Golding, S.; }
$$

Grillo, I. Equilibrium surface adsorption behaviour in complex anionic / nonionic surfactant mixtures, Langmuir, 2007, 23, 10140-10149 

Self-assembly in mixed dialkyl chain cationic - non-ionic surfactant mixtures, Langmuir, 2008, 24, 7674-7687

(21) Glen, K. M.; Moroze, S.; Bhattacharya, S. C.; Palepu, R. M. Effect of ethylene glycol on the thermodynamics and micellar properties of Tween20, 60 and 80, J. Disp. Sci. Technol. 2005, 26, 79-86 Preu, H.; Zradba, A.; Rast, S.; Kunz, W.; Hardy, E. H.; Zeidler, M. D. Small angle neutron scattering of $\mathrm{D}_{2} \mathrm{O}$-Brij35 and $\mathrm{D}_{2} \mathrm{O}$-alcohol-Brij35 solutions and their modelling using the P-Y integral equation, PCCP, 1999 , 1, 3321-3329

$$
\text { Song, S. S.; Hu, Z. G.; Wang, Q.; Lang, G. C.; Liu, X. H. Physicochemical }
$$
properties and surface tension predictions of mixed surfactant systems; Triton X-100 with $\mathrm{C}_{12} \mathrm{PyBr}$ and SDS, J. Disp. Sci. Technol. 2008, 29, 763-768 copolymer micelles delivered by SANS, Coll. Surf. A, 2003, 213, 175-187

$$
\text { Guo, L.; Colby, R. H.; Lin, M. Y.; Dado, G. P. Micelle structural changes in }
$$
aqueous mixtures of non-ionic surfactants, J. Rheol. 2001, 45, 1223-1243 Mortensen, K. Structural studies of aqueous solutions of PEO-PPO-PEO triblock copolymers, their micelle aggregation and mesophases: a small angle scattering study. $J$. Phys: Condens Matt, 1996, 8, A103-A124

Schwuger, M. J. Mechanisms of interaction between ionic surfactants and polyglycol ethers in water, J. Coll. Int. Sci. 1973, 43, 491-498

(28) Chai, L.; Goldberg, R,; Kampf, N,; Klein, J. Selective adsorption of PEO onto a charged surface mediated by alkali metal ions, Langmuir, 2008, 24, 1570-1576

(29) Heenan, R. K; Penfold, J; King, S.M, SANS at pulsed neutron sources: present and future prospects, J Appl Cryst, 1997, 30, 1140-1150

$$
\text { Heenan, R. K.; Rogers, S. E.; Turner, D.; Terry, A. E.; Tredgold, J.; King, S. M. }
$$
Small angle scattering using SANS2D, Neutron News, 2011, 22, 19-21 
Heenan, R. K.; King, S. M.; Osborn, R.; Stanley, H. B. RAL Internal Report, 1989, RAL-89-128

(32) Corbett, J. C. W.; Jack, R. O. Measuring protein mobility using modern microelectrophoresis, Coll Surf A, 2011, 376, 31-41

Viera, J. B.; Li, Z. X.; Thomas, R.K. Adsorption of triblock copolymers of ethylene oxide and propylene oxide at the air-water interface: the surface excess, J. Phys. Chem. B, 2002, 106, 5400-5407

Hayter, J. B.; Penfold, J. Determination of micelle structure and charge by small angle neutron scattering, Coll. Polym. Sci. 1983, 26, 1022-1030

Hansen, J. P.; Hayter, J. B. A rescaled MSA structure factor for dilute charged colloidal dispersions, Mol. Phys. 1982, 46, 651-656

Ashcroft, N. W.; Lekner, J. Structure and resistivity of liquid metals, Phys. Rev. 1966, $145,83-90$

Penfold, J. Neutron Scattering studies of micellar structure, Encylopedia of surface and colloid science, 2002, Marcel Dekker, NY, page 3653

(38) Tucker, I. M.; Petkov, J. T.; Jones, C.; Penfold, J.; Thomas, R. K.; Rogers, S. E.; Terry, A. E.; Heenan, R. K.; Grillo, I. Adsorption of polymer-surfactant mixtures at the oil-water interface, Langmuir, 2012, 28, 14974-14978

(39) Goldmints, I.; Yu, G. A.; Booth, C.; Smith, K. A.; Hatton, T. A. Structure of deuterated PEO - PPO - deuterated PEO block copolymer micelles as determined by SANS, Langmuir, 1999, 15, 1651-1656

(40) Hayter, J. B. A self-consistent theory of dressed micelles, Langmuir, 1992, 8, 2873-2876

(41) Pincus, P. Colloidal stabilisation with grafted polyelectrolytes, Macromolecules, 1991, 24, 2912-2919 

behaviour of pH-responsive poly-base brushes, Langmuir, 2007, 23, 5769-5778 Bergeron, V. Measurement of forces and structure between fluid interfaces, Curr. Opin. Coll. Int. Sci. 1999, 4, 249-255

(44) Bergeron, V.; Waltermo, A.; Claesson, P. M. Disjoining pressure measurements of foam films stabilised by nonionic sugar based surfactants, Langmuir, 1996, 12, 13361342

Xu, H.; Li, P. X.; Ma, K.; Thomas, R. K.; Penfold, J.; Lu, J. R. Limitations in the application of the Gibbs equation to anionic surfactants at the air-water interface: sodium dodecyl sulfate and sodium dodecylmonooxyethylene sulfate above and below the $\mathrm{cmc}$, Langmuir, 2013, 29, 9335-9351

(46) Lu, J. R.; Thomas, R. K.; Penfold, J. Surfactant layers at the air-water interface; structure and composition, Adv. Coll. Int. Sci. 2000, 84, 143-304

(47) Evans, D. F.; Mitchell, D. J.; Ninham, B. W. Ion binding and dressed micelles, J. Phys. Chem. 1984, 88, 6344-6348

(48) Kirby, B. J. Micro- and nano-scale fluid mechanics, transport in micro-fluidic devices, Cambridge University Press, 2010 
TABLE of CONTENT GRAPHIC

\section{On the nature of the inter-micellar interactions in ethoxylated polysorbate surfactants with high degrees of ethoxylation}

J. Penfold, R.K. Thomas, P. X. Li, I. Tucker, J. Petkov, R E Petkova

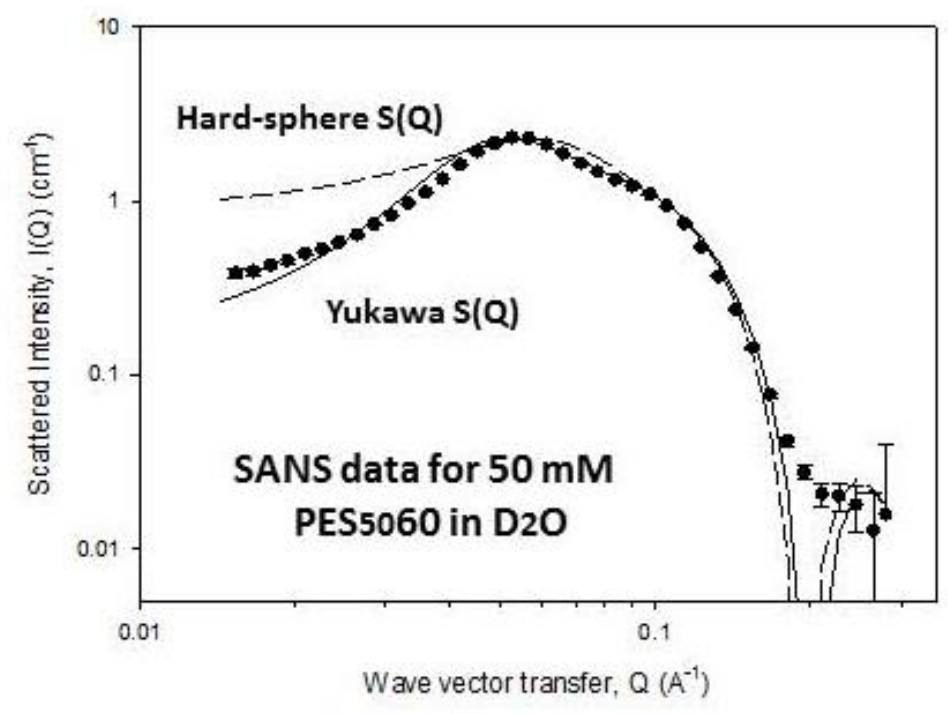

